\title{
Scalagna 0.1: Towards Multi-tier Programming with Scala and Scala.js
}

\author{
Bob Reynders \\ imec-DistriNet, KU Leuven \\ Leuven, Belgium \\ bob.reynders@cs.kuleuven.be \\ Dominique Devriese \\ imec-DistriNet, KU Leuven \\ Leuven, Belgium \\ dominique.devriese@cs.kuleuven.be
}

\author{
Michael Greefs \\ imec-DistriNet, KU Leuven \\ Leuven, Belgium \\ greefs.michael@hotmail.com \\ Frank Piessens \\ imec-DistriNet, KU Leuven \\ Leuven, Belgium \\ frank.piessens@cs.kuleuven.be
}

\begin{abstract}
In the state-of-practice, developing web applications requires dealing with multiple programming languages or codebases. To address this issue, researchers have proposed multi-tier languages such as Hop or Links that support client and server development in a single language and in one codebase. Even if such multi-tier languages are often strongly based on an existing language - for instance Hop is based on Scheme - they are new languages, and require a new compiler.

The objective of this paper is to define a multi-tier language as a library-based Scala DSL. Scala already supports compilation to both the Java VM and to JavaScript. The multi-tier language we propose in this paper, Scalagna, combines the existing Scala JVM and JavaScript ecosystems into a single programming model without requiring changes to, or rewrites of the existing Scala compilers. We discuss how this is possible using Scala's excellent support for definining DSLs, and the experimental Scala macro system. We show that Scalagna has reasonable performance, and by porting an existing Eliom application, we provide evidence that Scalagna is as expressive as other established and existing multi-tier languages.
\end{abstract}

\section{CCS CONCEPTS}

-Software and its engineering $\rightarrow$ Domain specific languages;

\section{KEYWORDS}

Scala, multi-tier, web programming

ACM Reference Format:

Bob Reynders, Michael Greefs, Dominique Devriese, and Frank Piessens. 2018. Scalagna 0.1: Towards Multi-tier Programming with Scala and Scala.js.

Permission to make digital or hard copies of all or part of this work for personal or classroom use is granted without fee provided that copies are not made or distributed for profit or commercial advantage and that copies bear this notice and the full citation on the first page. Copyrights for components of this work owned by others than ACM must be honored. Abstracting with credit is permitted. To copy otherwise, or republish, to post on servers or to redistribute to lists, requires prior specific permission and/or a fee. Request permissions from permissions@acm.org.

<Programming'18> Companion, April 9-12, 2018, Nice, France

(c) 2018 Association for Computing Machinery.

ACM ISBN 978-1-4503-5513-1/18/04 ..\$15.00

https://doi.org/10.1145/3191697.3191731
In Proceedings of 2nd International Conference on the Art, Science, and Engineering of Programming (<Programming'18> Companion). ACM, New York, NY, USA, 6 pages. https://doi.org/10.1145/3191697.3191731

\section{INTRODUCTION}

Developing web applications presents a number of interesting challenges for programmers. One challenge is due to the inherent distributed nature of the platform with parts of the application running on the server and other parts on the (zero or more) clients. This causes an impedance mismatch within applications, for example, keeping client and server programming interfaces synchronized is difficult and tedious. Multi-tier programming languages [see among others $1,5,8$ ] aim to resolve this problem by allowing both the client and server parts of a web application to be written in a single language and codebase. These existing multi-tier languages are new programming languages requiring a new compiler, and hence a significant initial development cost.

Our objective in this paper is to investigate whether we can get the benefits of a multi-tier language without the cost of having to develop a new compiler. More specifically, we aim to implement a Domain Specific Language (DSL) for multi-tier programming as a Scala library, without the need for a new compiler (or changes to existing compilers). We start from Scala, because it already has all the key components. Scala is a language with a healthy amount of libraries. Its primary runtime is the JVM and it boasts fluent Java interoperability. In addition, Scala.js is a plugin that compiles Scala to JavaScript with strong focus on interoperability with existing ecosystems [3].

The key contributions of this paper are:

- We design and implement Scalagna, a multi-tier programming environment for Scala based on the existing JavaScript and JVM ecosystems that requires no additional compiler changes or plugins. It uses Scala's experimental macros to do minor rewrites as explained in section 4 .

Scalagna is publicly available at https://github.com/tzbob/ scalagna.

- We quantify the performance cost of Scalagna by comparing the performance of a Scalagna application with the same application developed directly in Scala.

- We evaluate the expressiveness and verbosity of Scalagna through a comparison with Eliom, an established multi-tier language extension to OCaml. 


\section{SCALAGNA BY EXAMPLE}

Scalagna web programs are written in a single codebase. Two programs are extracted, one program runs on the web-server while the other runs on the browser. In this section we give you a feel of what it would be like to program with Scalagna before we go into the nitty-gritty of the system. We focus on features and solutions that are needed in a modern client/server web application.

\subsection{Pages \& Annotations}

In Scalagna, you can write client and server code within the same codebase. Annotations are used to mark the destination of a code block. For example, if we want to create a page to print two statements, on the client and server respectively:

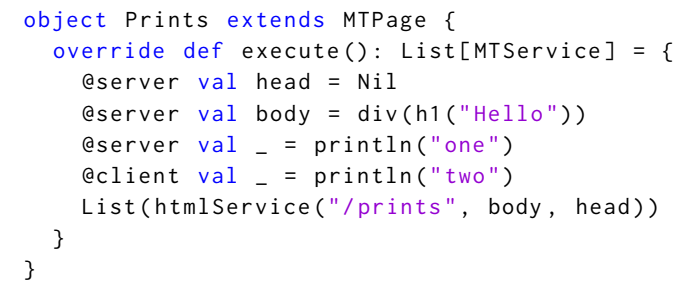

A page is defined as a list of services and in this small example we use the HTML service. An HTML service is defined by a path relative to the web server, a body and the head. Both body and head are server values, they represent the HTML content of the served page, in this case, a simple hello title and an empty head.

The print statements are added as annotated nameless values, depending on the annotation the evaluation of the value is server or client side. In this case, as the server starts up, "one" is printed on the server console. Every time a user accesses the "/prints" page on his browser, "two" is printed in the browser's console.

\subsection{Exposing Server State}

While the previous example showed us how to execute initial sideeffects for both the client and server tiers, it did not hint at how we could deal with typical server state. For example, what does a visitor counter look like in Scalagna?

In Scala, mutable and immutable references are explicitly denoted with the val and var keyword. In the previous example we annotated immutable values, but Scalagna also supports the client and server annotation of variables as well as methods (def) and type aliases (type).

In Figure 1, a mutable variable counter represents the visitor count. Access to this mutable server variable is available through . value on counter. The body of the page displays a string representation of the counter. Note it is now defined as a method instead of a value, and the counter is increased every time the body is evaluated. Since Scalagna HTML services re-evaluate body and head on each serve to a client, a new client updates the value of the counter, and the new value is embedded in the page.

While Figure 1 shows how to expose server state to the client through HTML, it does not provide a direct solution to using server values in clients, e.g., having a client-side counter. In Figure 2, we demonstrate the injection feature. Server values can be injected into client contexts, injected values are added into a client's context every time a user accesses a page. The example behaves as the first

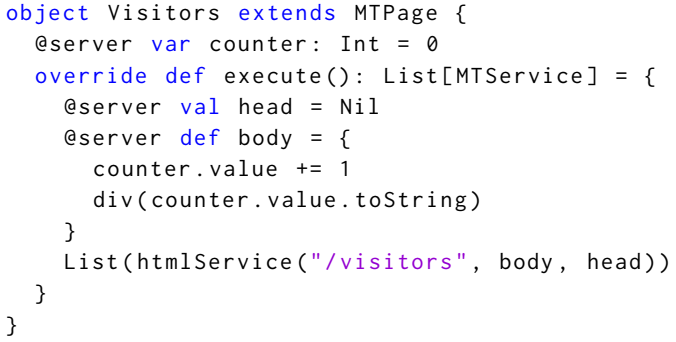

\section{Figure 1: Visitor Counter}

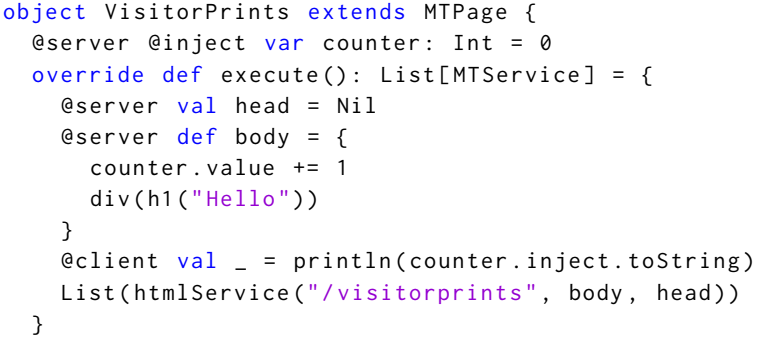

Figure 2: Visitor Counter Prints

example we showed above. Every time a user accesses the page the browser's console will show the current visitor count.

\subsection{Dynamic Client/Server Interaction}

So far, interactions between client and server have been static. Client values are injected and HTML values are determined when a user requests a page. Modern web applications require more flexibility than this. It is beneficial to the user experience if client code can interact with the server during its execution, it makes interactive applications such as chats possible without reloading pages. These interactions are first-class values in Scalagna. They are added as services.

In Figure 3, we show an example that uses such a service. The application ignores all security advice and allows direct access to the file system from the browser by exposing an RPC that can read files. The rpcFileRead service is created from a string that represents the path on which it will be made available and a server function. This server function will be executed when the service is used, its results are serialized and sent back to the client. In Figure 3, on line 14, the service is used. It can be called as a regular function, however, its return type is wrapped in a Future, i.e., a value to denote an asynchronous operation. With foreach, the success case ${ }^{1}$ of the asynchronous operation is handled and our file contents are printed to console.

With these features combined, we have a minimal but powerful way of expressing web applications as one application. We discuss more features in the next section and a larger example that uses all

\footnotetext{
${ }^{1}$ We ignore the error case in this contrived example.
} 


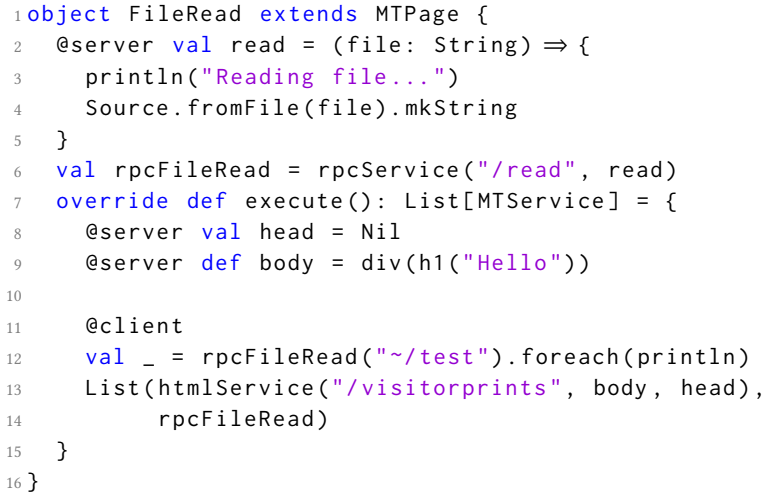

\section{Figure 3: File Read}

of our features when we compare Scalagna to Eliom [5] in section 5 on evaluation.

\section{API}

The examples did not cover the entire Scalagna API. In this section we briefly look at each feature and its types in detail. We largely follow the API of Eliom, making changes where it decreases added complexity to Scala/Scala.js.

Bootstrapping. Scalagna programs are defined through a list of pages, which are defined through a list of services:

trait MTApp \{def mtMain: List[MTPage] \}

trait MTPage \{ def execute: List[MTService]

Client/Server. Programmers make use of the @client and @server annotations to mark which sections of the code belong to which tier. Any code that is not marked with such an annotation belongs to both tiers. Note that shared variables are not synced across tiers, they are duplicated. Changing shared variables' value does not impact their value on other tiers automatically and their use is not recommended.

Each annotation turns a value into its respective tier value: @client val $\mathrm{x}$ : Int $=1$ becomes a value $\mathrm{x}$ of type MTClient [Int], likewise for the server counterpart. Variables turn into MTClientVariable s. The API of these tier values is the following:

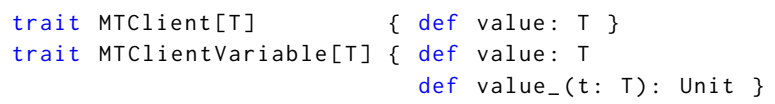

The value accesses the client value. If this method is used in a client context it returns the expected value. If the method is used in a server context instead, it fails with a compile-time error, letting the programmer know that that particular value is not available in that tier. Note the extra method definition on the variable - in Scala this enables the setter syntax so that assignment is possible, e.g., $x$. value $=5$. The server values and variables behave the same.

Annotations can also be put on methods and type aliases. An annotation on a method rewrites its return type, e.g.: @server def $x()$ : T turns into def $x()$ : MTServer[T]. Type aliases are even simpler, they are erased from the opposite tier.
Injection. It is possible to make server values available in a client context without paying a large cost. Injecting a server value into a client value is done when a page is loaded. Upon load time the current value of the to-be-injected server value is added to the initial payload. Later, an initialization phase in the client program retrieves these injected values. The need for these kind of injections is high when developing client/server web applications. Serverbased "initial" values are required all the time, the history of a chat box, an initial playing field of a game, etc.

To inject a server value it first has to be marked as being injectable through an annotation:

@server @inject val init: Int $=0$

@client def return = println(init. inject)

An extra requirement for injections is the presence of a type declaration. This type is used to derive the (de)serialization mechanics ${ }^{2}$.

Fragments. Fragments is something we have not yet touched upon. A fragment is a special type of client value that has meaning on the server. Its use in Scalagna is limited to binding Scala.js functions to server-rendered HTML. This supports convenient generation of HTML pages that use the tag attribute event binding, e.g., <button onclick=...>...

An HTML fragment is created by adding an additional annotation to a client value:

@client @fragment val onclk $=(e l, e) \Rightarrow \operatorname{println}(e l, e)$

The only fragments that are supported right now are functions of the following type:

(el: dom.Element, e: dom.Event) $\Rightarrow$ Unit

They take the bound DOM element and an event as input and return nothing. Scalagna supports using fragments in an existing Scala HTML DSL ${ }^{3}$, e.g.:

@server val body = button (onclick:= onclk)

Services. All services that are available follow the same usage pattern. Anything needed to define the service itself is required in its creation function and all functionality that it provides is available through the service value itself:

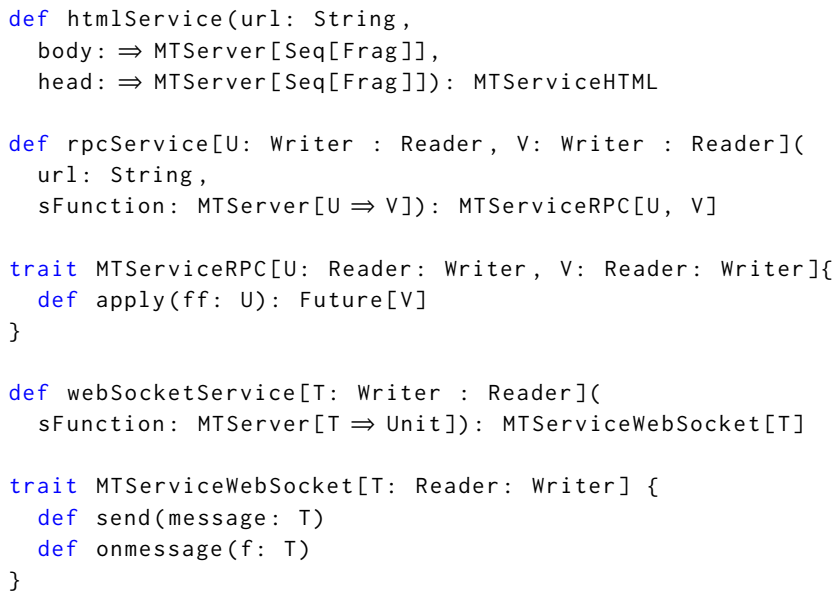

${ }^{2}$ Note that no custom serialization code was written for Scalagna, it integrates with typical Scala generic serialization libraries that use a combination of implicits and macros.

${ }^{3}$ https://github.com/lihaoyi/scalatags 
Regarding HTML, in this DSL HTML tags are of type Frag. The MTServiceHTML that is returned is not used for anything but registering the service.

The MTServerRPC service is created from a path and from a server function to define its behavior. Its value is used to make the RPC call in a client context.

The websocket service requires a server function to define the server-side reaction. Its return value can be used to send values and listen to messages on the client side. Note that our service at the moment does not allow the server to initiate messages, instead it acts as a broadcast channel. All client messages are forwarded to all other clients. However, this is purely due to time constraints and there is no technical limitation stopping us from exposing the full websocket API.

\section{IMPLEMENTATION}

Due to the limited space we can only provide a high-level overview of the implementation, both the implementation as well as some examples are available online ${ }^{4}$.

As mentioned during the introduction, one of the key design goals of Scalagna was to lift the Scala.js and Scala ecosystem into a multi-tier programming environment with as little additional complexity as possible.

Scalagna is completely library based. It does not require a compiler plugin or changes to Scala.js. It relies on structuring the program in a specific way and uses macros to increase compatibility with existing ecosystems.

\subsection{Structure}

A Scalagna application has one main method for a user to fill in, one API to create an HTML service, etc., but the same codebase does vastly different things on the client or the server. In practice, a Scalagna application is one codebase used to derive two applications, a JVM application that runs a standard Scala web server and a Scala.js application. The entire structure is shown in Figure 4.

The Scalagna codebase is split into three parts, a shared, JVM and a JS part. This is a typical way of writing cross-platform Scala projects, the shared section of the codebase, as the name implies, contains all code that is shared between client and server. The JVM and JS parts can both use the shared section of the codebase but not each other. The Scalagna library is structured so that the API definitions lives in the shared section and tier-specific implementations of them are in the corresponding JS/JVM sections.

For example, in Figure 4, we show two implementations of MTApp. One starts a web server and handles serving HTML pages, replying to RPC actions, etc., the other handles initialization of clients e.g., injected (server $\Rightarrow$ client) values. The same structure is used to implement pages and services.

The implementations of MTPage and the various services work similarly. The JVM implementations of those APIs register the required functionality with the web server while the JS implementations of those APIs do the appropriate calls when needed, e.g., an RPC service is registered on the JVM while the JS version makes calls to the server functionality.

\footnotetext{
${ }^{4}$ https://github.com/tzbob/scalagna
}

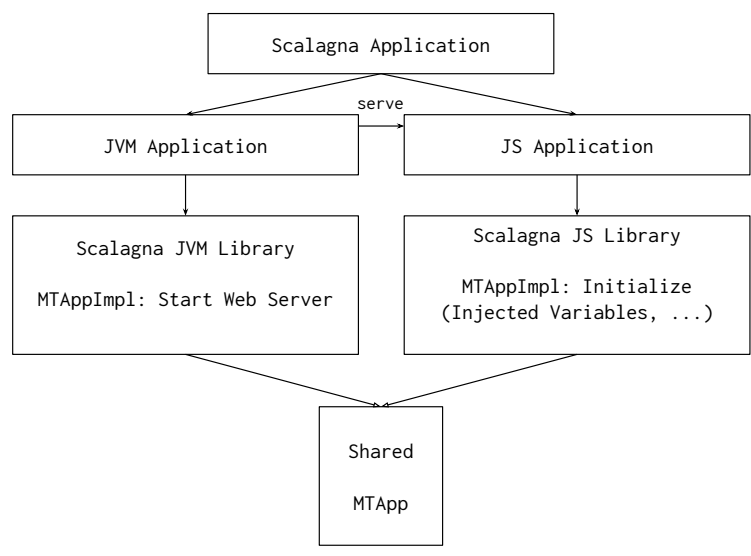

Figure 4: Scalagna Structure

\subsection{Annotations}

The implementation of the annotations in Scalagna are much less complicated than they seem. They are macro based but they do not do any sophisticated checks, they are simple wrappings on the AST. For example, annotating a value with @client causes the client macro to wrap the value to an internal method call. This method call consists of regular, non-macro code, that wraps the value in a MTClient value.

So far this did not require the power of macros, a client method could have done this. However, reusing the Scala.js ecosystem becomes problematic:

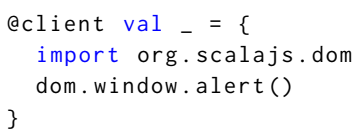

In this example, the compilation fails on the server since there is no JVM library to supply the package org. scalajs. dom. This is strictly a Scala.js library. We solve this problem in the server version of the @client macro by erasing the client-annotated code before the Scala compiler tries to compile it. As such, the typechecked version of the codebase on the server no longer has the clientside import. This allows Scalagna to support ecosystem-specific libraries in annotated sections. Instead of the removed code, the macro inserts a dummy MTClient on which . value is not a valid call. This follows the same approach as we explained above, MTClient has two implementations, one for server and client.

We do not allow the nesting of macros in Scalagna and for technical reasons require types on injected server values which we also check in the macros. However, the code for an entire @client macro is 130 lines of code with a large amount being static definitions of ASTs.

\section{EVALUATION}

The following microbenchmarks are executed on a Windows 10 laptop using an i7-2720QM CPU with the Chrome browser.

Server Performance. The server side of Scalagna makes use of http $4 s^{5}$, a Scala web server. In our first micro benchmark we test a

$\overline{5_{\text {http://http4s.org/ }}}$ 


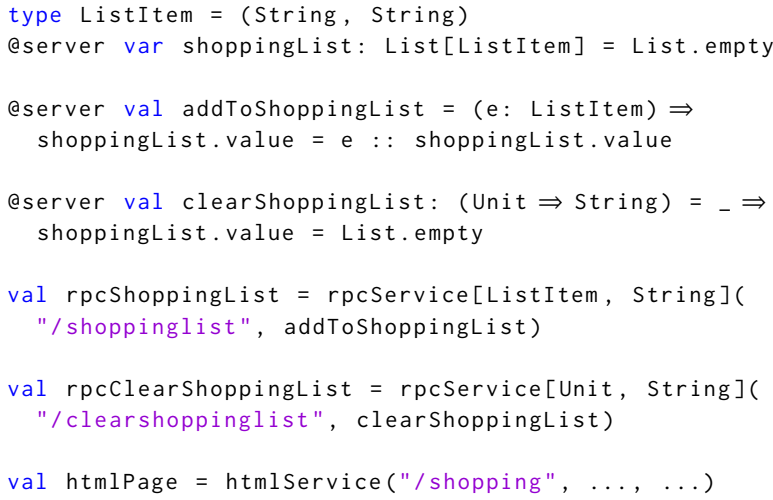

Figure 5: Shopping List

Table 1: Server Performance

\begin{tabular}{c|c|c|c} 
& \# req & average(ms) & req/sec \\
\hline Scala & 78812 & 12 & 263 \\
Scalagna & 77858 & 12 & 260
\end{tabular}

shopping list application. The server section of this application can be seen in Figure 5. There are three services, one serves an HTML page, one clears the internal list and one adds a value to the internal list. In our benchmark we simulate 2000 users using Locust ${ }^{6}$. In Table 1, we show our results. As expected, they are really close, the small difference can be because of the way requests are matched but the differences are so small it could just be benchmark noise.

Client Performance. We test the client overhead by comparing a Scala.js application to the client side of a Scalagna application. The program that we benchmark is the client side of the shopping list application. We measure the overall code size and the time it takes to execute the main function, that is, perform the initialization of the application.

In Table 2, we show the impact Scalagna has on page load times. We use the Google Chrome Devtools and separate our measurements into two pieces, the time it takes to parse and execute the script and the time it takes to execute the main function. For handwritten Scala.js code the initial loading time is higher because the code is handwritten for the problem and no injection is used to get values from the server into Scala.js. Contrary to Scalagna, where injections are used and the initial executing of the script does barely anything.

The second phase is more interesting, the handwritten code has no initialization logic so the time to call main is negligibly low. Scalagna on the other hand performs two operations, it first looks through all the pages in the Scalagna program to initialize the current page, after which the selected page gets its appropriate injected values initialized. In our version 0.1 this code is written without performance in mind and is relatively slow, however, at this point the user already has the full HTML rendered and the

\footnotetext{
${ }^{6}$ https://locust.io/
}

Table 2: Client Performance (ms)

\begin{tabular}{c|c|c|c} 
& full-opt.js & shopping.main() & Total \\
\hline Scala.js & 59 & 0.4 & 59.04 \\
Scalagna & 51 & 41 & 92
\end{tabular}

interactivity of the application is delayed by only $40 \mathrm{~ms}$. This overhead seems acceptable, and there are significant opportunities for further performance improvements.

Practical Comparison with Eliom. As a test of Scalagna's expressiveness and verbosity we make a direct comparison with Eliom, a multi-tier language based on OCaml. Eliom provides a step-bystep tutorial to learn its features based on incrementally extending an example. In this example programmers build a live multi-user Graffiti application where users can collaborate on a canvas.

Both applications make use of HTML and push-services (web sockets or comet implementations), HTML DSLs, RPC calls, injections and fragments. We lack the space to make a direct comparison of the applications in the paper, but we can show a small excerpt in Figure 6 and 7 instead. The full source of the example is available on the project page ${ }^{7}$.

We compare with the code from the Eliom tutorial application ${ }^{8}$, up to "Sending the initial image" at 115 lines of code. Our source finishes at 140 lines of code formatted using a production realistic formatting ( 80 characters-wide). However, Scala is a braces language, and without trailing braces the code is only 124 lines long. The rest of the difference is related to not having better import features, requiring more boilerplate to setup the application (implementing MTPage), etc.

This comparison is shallow but we do feel that it gives us enough confidence to say that we have enough features to create impressive web applications without having the codebase inherently suffer from great overhead.

\section{RELATED WORK}

We can divide multi-tier languages into two large categories. The first are those that are built from scratch such as Ur/Web [1] or Links [2]. Such languages can be tailored to the domain of web programming. For example, Ur/Web closely ties its garbage collection to HTTP requests. However, these languages start out with a fresh ecosystem and often lack the rich library that existing languages have to offer.

The second category of multi-tier languages are based on existing languages. Hop [8], is based on the Scheme language. It is made up of two compilers: one compiles code executed by the server, the other compiles code executed by the client. While Scheme has existing libraries, it's not the most popular language with web developers. Hop's successor, Hop.js [7], aims to solve this by using JavaScript as its main language. While still being run through its own compiler to allow server parallelism, the project also focuses on compatibility with existing Node.js libraries. In Hop.js, services are created using their own keyword in the language and compared to

\footnotetext{
${ }^{7}$ https://github.com/tzbob/scalagna

${ }^{8}$ https://ocsigen.org/tuto/manual/application

${ }^{9}$ The last extension does a server-side image rendering, the server libraries used would be too different to make a good comparison.
} 


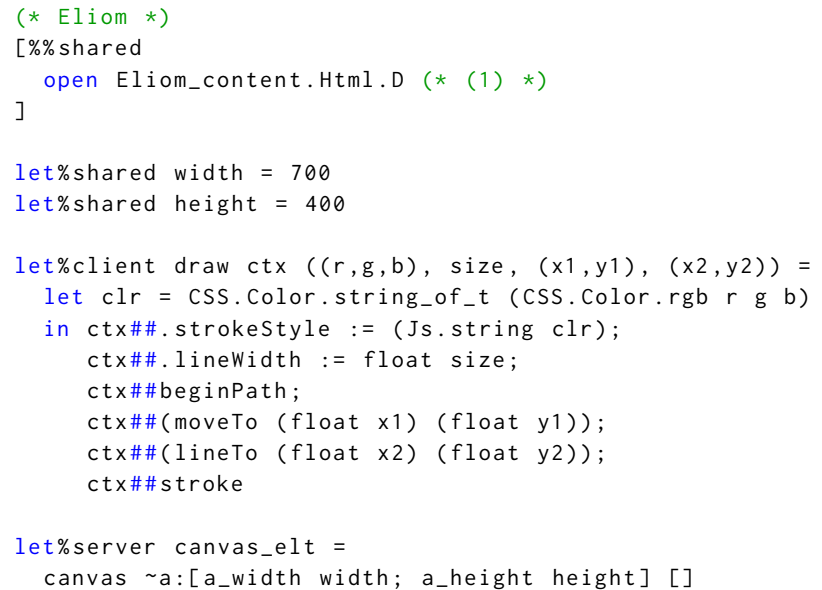

Figure 6: Eliom Draw

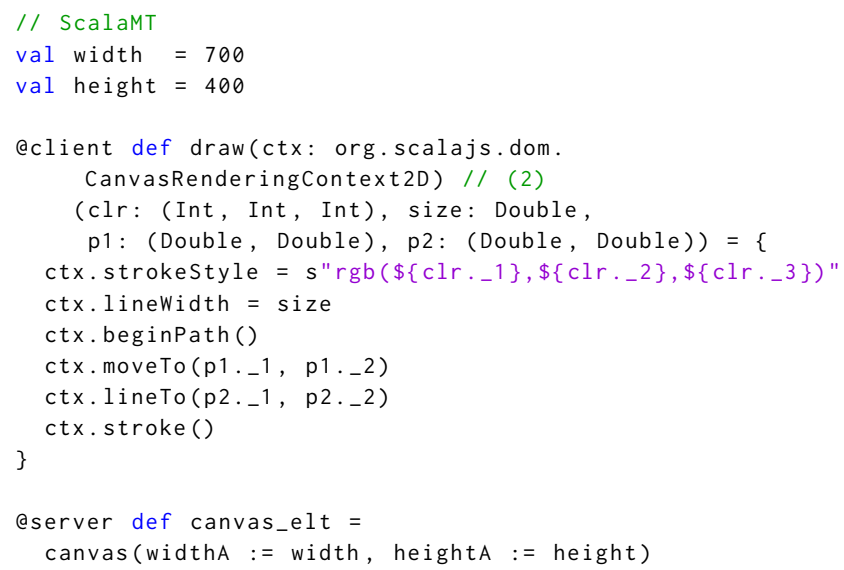

Figure 7: Scalagna Draw

our limited use of fragments it allows true full stage programming to define client programs within other client or server programs.

Eliom [5] is based on OCaml and its semantics were a big inspiration to all the decisions made in Scalagna. Its features are very similar, services are created through functions and client and server sections are annotated per declaration. It is more mature than Scalagna and has a better theoretic foundation. Its fragments are truly server values, e.g., a fragment function can be applied to a fragment parameter.

Haste [4] is based on Haskell, the developers created a GHC to JavaScript compiler and provide a library approach to multi-tier programming. It has no need for client or server annotations. It uses a client and server monad, since there are no untracked side-effects all pure code can be compiled for both versions of the program. The server monad is executed server-side and the client monad defines the client program. It has the issue of not being able to effectively deal with libraries that only exist in the ecosystem on one tier.

JS-Scala [6] is a Scala DSL to write embedded JavaScript programs. It allows multi-tier programming by treating the Scala program as a web-server and a program generator. However, its support for JavaScript and Scala library interop is much less than Scala.js.

In industry the Meteor ${ }^{10}$ framework gained popularity. It uses JS on both client and server. Code is "annotated" by checking a variable isserver or by separating files into a client or server directory.

\section{ACKNOWLEDGMENTS}

Bob Reynders holds an SB fellowship of the Research Foundation Flanders (FWO). Dominique Devriese holds a postdoctoral fellowship of the Research Foundation - Flanders (FWO). This research is partially funded by the Research Fund KU Leuven, and by the SBO-project TearLess.

\section{REFERENCES}

[1] A. Chlipala. Ur/Web: A Simple Model for Programming the Web. In POPL, pages 153-165, 2015.

[2] E. Cooper, S. Lindley, P. Wadler, and J. Yallop. Links: Web Programming Without Tiers. FMCO, pages 266-296. 2007.

[3] S. Doeraene, T. Schlatter, and N. Stucki. Semantics-driven interoperability between Scala. js and JavaScript. In SCALA, pages 85-94, 2016.

[4] A. Ekblad and K. Claessen. A seamless, client-centric programming model for type safe web applications. In Haskell, pages 79-89, 2015.

[5] G. Radanne, J. Vouillon, and V. Balat. Eliom: A core ML language for Tierless Web programming. In APLAS, pages 377-397, 2016.

[6] J. Richard-Foy, O. Barais, and J.-M. Jézéquel. Efficient high-level abstractions for web programming. In GPCE, pages 53-60, 2013.

[7] M. Serrano and V. Prunet. A Glimpse of Hopjs. In ICFP, pages 180-192, 2016

[8] M. Serrano and C. Queinnec. A multi-tier semantics for Hop. High-Order Symb. Comput., 23(4):409-431, 2010

\footnotetext{
$\overline{{ }^{10} \mathrm{https}: / / w w w . m e t e o r . c o m / ~}$
} 